\title{
Surrogate Variable
}

National Cancer Institute

\section{Source}

National Cancer Institute. Surrogate Variable. NCI Thesaurus. Code C142725.

A factor or condition subject to change that provides an indirect measurement of effect

in situations where direct measurement of clinical effect is not feasible or practical. (ICH) 